\title{
Deep Electroosmosis Technology for Oil Fields
}

\author{
E.G. Porsev \\ Novosibirsk State Technical University \\ Novosibirsk, Russian Federation \\ e-mail: porseve@ngs.ru
}

\author{
B.V. Malozyomov \\ Novosibirsk State Technical University \\ Novosibirsk, Russian Federation \\ e-mail: mbv5@mail.ru
}

\begin{abstract}
The problem of reclamation of waste pits in oil fields is still being solved by a costly method. The accelerated technology of sludge dewatering with electroosmotic effect is proposed. The concept of specific energy inputs for mass transfer is introduced. To eliminate the influence of relaxation of gradient fields, it is proposed to change the parameters of electric energy.
\end{abstract}

Keywords - oil fields, waste pit, electroosmosis, reclamation of waste pits, relaxation of force gradient fields.

\section{INTRODUCTION}

Waste pits are pits about 50-100 $\mathrm{m}$ in diameter, sometimes smaller. They are created near boreholes, for storing drilling waste: waste solutions, with or without oil, crushed rock, clay, water, various chemical reagents. In each pit storing, there are about 500 cubic meters of drilling waste per well. Drilling waste, diluted with water, is a viscous creamy substance, to some extent is toxic.

In order to eliminate this danger to the environment, waste pits are recultivated in well construction. There are several ways of recultivation: filling with sand, natural evaporation, thermal and chemical treatment, pumping into dry absorbing layers, "squeezing out" into narrow trenches, export to evaporation fields. All of them are very expensive, both in time and in finance.

To fill one pit, one needs to bring 4-5 thousand cubic meters of sand, spending a lot of money for all the work to eradicate the pit. At the same time, to bring sand from other places, one must destroy the forest and from under it, take the sand or get it from the lake or swamp.

The purpose of the work from the point of view of the criterion of public utility is to accelerate the process of reclamation of waste pits, to save primary energy producers in the process, and improve the ecological safety of oilfields. tasks: a) to find the optimal way of sludge dewatering;

b) to calculate the technological cycle of the reclamation system on a new basis;

c) to develop technology schematic diagrams and an electric scheme for electroosmotic recultivation of oilfield sludge barns.

Analysis of the patent situation in the field of remediation technology for the period from 1975 to 2008 to determine the trend in the development of technology showed (Fig. 1):

1) The distribution of the frequency of the repetition of the filing of applications by years in the study period is uniform, which indicates that the problem of reclamation is unresolved.

2) An analysis of the frequency of repetition of filing patent applications during the period under review indicates that the problem has not been resolved, and a slight increase in the number of applications in recent years indicates an increase in the relevance of technical solutions related to the remediation of lands by technical means.

3) Obviously, the problematic situation is that in this area there are no radical revolutionary solutions that would help solve the problem in traditional ways.

4) A working hypothesis is proposed for the possibility of solving the above-mentioned problem by an electrotechnical method, using electro-osmotic water reduction.

Based on the analysis of the patent situation, it can be said that to date, electroosmosis for dewatering of slurry barns has not been used, despite the wide application of this technology in other areas of technology. In the authors' opinion, the reason for the difficulty is in predicting the result and lack of practical recommendations, combined in one histogram and continued until 2015.

At the same time, electroosmosis allows not only lowering the moisture content of the dispersed material, but also, together with moisture, removing hardness salts from 
Quantity, ones 4

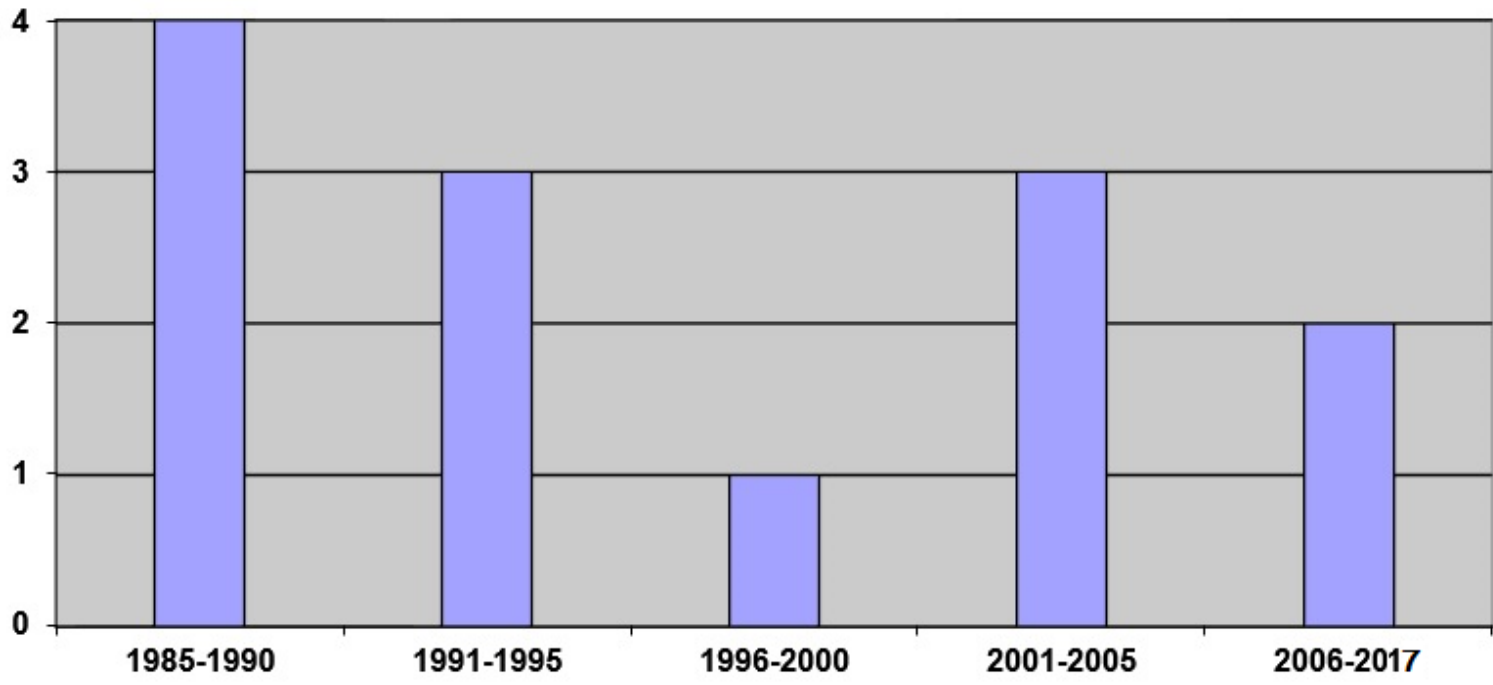

Fig. 1. Histogram of the number of patents received and applications filed by years.

Quantity, ones

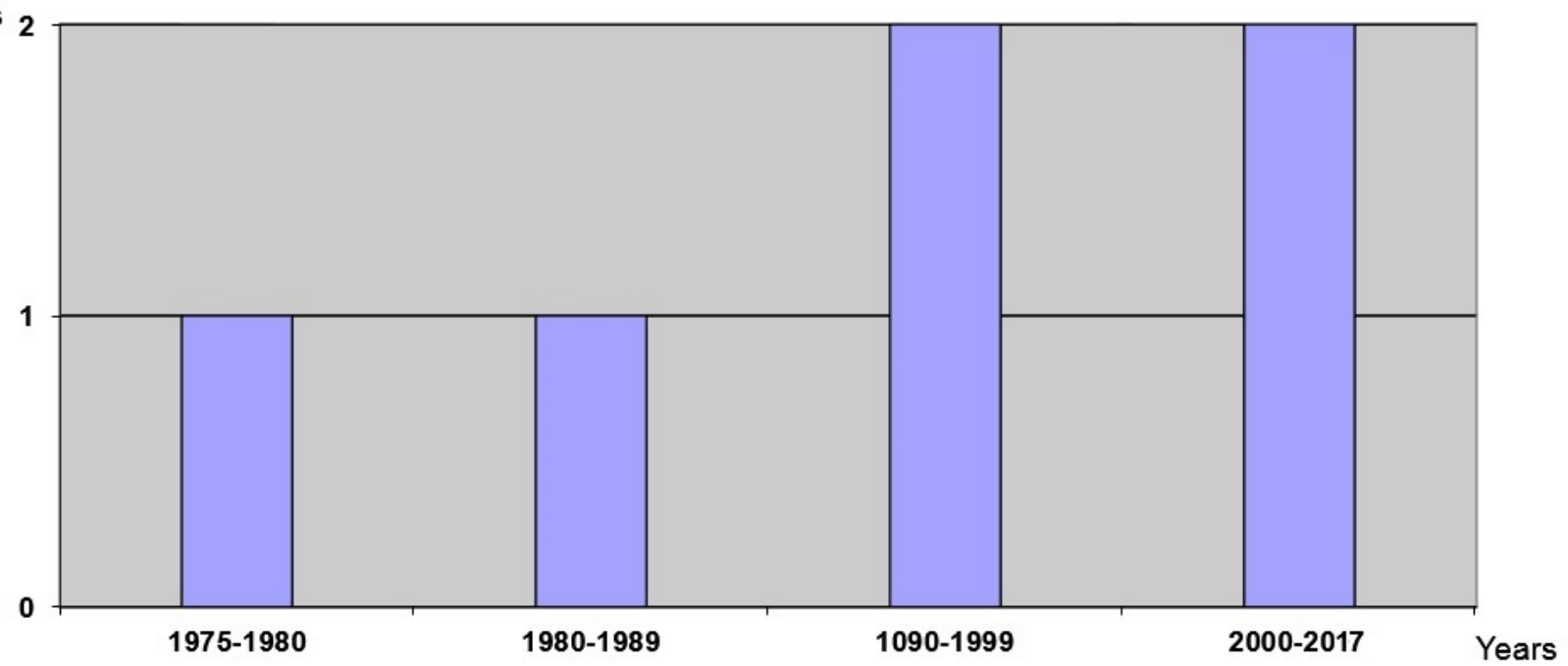

Fig. 2. Histogram of the number of published scientific papers by years.

it, which also contributes to the acceleration of the reclamation process.

\section{THEORY}

The first quantitative studies of electroosmosis were carried out by Wiedemann in 1852 . He showed that the volumetric velocity $V$ - electroosmosis - is proportional to the current strength $I$ with other fixed parameters $V / I$, and the ratio does not depend on the cross-sectional area and the thickness of the diaphragm [1]. The first quantitative characteristic of the process was the volumetric velocity, which shows Lomiz [2], the expenditure of electroosmotically transferred water, referred to the unit of the total section of the dehydrated material occupied by the skeleton and pores. Speed is expressed in meters per second. With the deepening of knowledge in the field of colloid chemistry and dispersed systems, $\xi$ - the potential [3] (Helmholtz and Perrin), measured in volts (B), was taken as the main indicator of the electrokinetic properties of the material. This indicator reflects the essence of physical phenomena, but in practice, it is inconvenient, since for its definition one requires precise measurements of the dielectric constant and viscosity.

In 1940, B.F. Reltov proposed the introduction of a new indicator - the coefficient of electroosmosis $K_{e}-$ to determine the efficiency of the process [4]. The coefficient of electroosmosis determines the effect of the solid and liquid components on the volumetric rate of electroosmosis and is equal to the fictitious velocity of water movement under the action of an external electric field at field strength $E, V / c m$. The value of the coefficient is defined as:

$$
K_{e}=\frac{\rho \xi \varepsilon}{4 \pi \eta}
$$


where $K_{e}$ is, firstly, the coefficient of proportionality between $V_{e}$ and $E$, and, secondly, - the characteristic of the integral:

$$
V_{e}=K_{e} \cdot E .
$$

At the same time, it is an auxiliary characteristic for determining the volumetric velocity. Further development was obtained in the coefficient of electroosmosis with respect to the current $K_{e i}-\left(\mathrm{m}^{3} / \mathrm{A} \cdot \mathrm{sec}\right)$, proposed by Zangirov [5-6]. In contrast, $K_{e}$ - the coefficient of proportionality between $V_{e}$ - the volumetric velocity and $\sigma-$ the current density:

$$
V_{e}=K_{e i} \cdot \sigma .
$$

The coefficient of electroosmosis is related to the coefficient of current electroosmosis by the dependence:

$$
K_{e}=\gamma \cdot K_{e i},
$$

where $\gamma-$ the specific electrical conductivity of the processed material, $\mathrm{Ohm} \mathrm{m}^{-1} \cdot \mathrm{m}^{-1}$. Both of these factors make it possible to designate the liquid output when working at DC and the power consumption in ampereseconds when designing by specifying the required volume of water. But when working on alternating or alternating asymmetric currents, this is difficult.

In connection with the need to work on an alternating asymmetric current, the need for an energy evaluation of the process, not with traditional coefficients $K_{e i}$ and $V_{e}$, but with a characteristic that takes into account all the energy expended, arose. It was suggested estimating the energy efficiency in the specific energy consumption for the release of liquid:

$$
w_{e}=\frac{W}{M}, k W h / k g \text {, }
$$

where $W-$ the amount of electricity expended on the dehydration process, $k W h ; M-$ mass of water, extracted by electroosmotic pressure, $k g$.

It is also possible to estimate the specific power:

$$
P_{e}=\frac{w_{\ni}}{\tau}=\frac{W}{M \cdot \tau}, k W / k g
$$

where $\tau$ - the processing time, hour.

Lokart [7-9] later adopted the same system for assessing the energy of the process. The advantage of the authors' evaluation system is that knowing the specific energy inputs and specifying the required depth of dehydration and processing time, it is easy to calculate the required power and operating current of the installation. Obviously, in each case, an experimental determination of the specific energy consumption is required.

Due to the fact that mineral particles are present as a dispersed phase of soil in almost all dispersed soil materials, and the water as the dispersion medium, the process of electrokinetic energy and mass transfer in them has its own peculiarities, namely:

A) Heteroporosity of the disperse phase, that is, the inhomogeneity of the capillary sizes, which depends on the large scatter of the particle sizes, which leads to an overuse of electrical energy for heating the dispersion medium in large capillaries.

B) The multicomponent nature of the electrolyte composition of the dispersion medium, that is, the presence of indifferent and non-indifferent electrolytes in the solution, leading, on the one hand, to a decrease in the electrochemical potential as a result of an increase in the concentration of counterions with an increase in the concentration of indifferent electrolytes and, on the other hand, to complete the crystal lattice of the dispersed phase, leading ultimately to a drop in the electrokinetic potential.

C) The difference between the hydrogen index of the medium and the $p H$ of the neutral can strongly affect the electro-kinetic potential of dispersed particles, since "hydrogen and hydroxyl ions have a high ability to adsorb; first - due to the small radius, which allows them to approach the surface of the solid phase closely; second because of the large dipole moment" [10-13]. The deviation of the water indicator to the acidic or alkaline side for the dispersive medium of the soil is a characteristic.

D) Dependence on the concentration of the dispersed phase - when diluting the colloidal system, the electrokinetic potential should increase, since the thickness of the double electrical layer increases as a result of the loss of the counterion concentration in the solution. At the same time, when diluting, the desorption of the potential-determining ion can be observed from the surface of the dispersed phase, which should lead to a drop in the electrokinetic potential. The phenomenon is typical for electrophoresis in highly diluted sewage waters.

E) Dependence on the treatment temperature - with increasing temperature, the $\xi$-potential must grow due to the increase in the intensity of the thermal motion of counter ions and an increase in the thickness of the double electric layer, but at the same time the desorption of the potentialdetermining ions can also increase, and at the same time, the $\xi$-potential decreases.

F) The instability of the electrophysical and electrochemical properties of the disperse phase is deduced logically from the physical nature of the particles of the dispersed phase and the chemical activity of the dispersion medium.

Thus, the main feature of the process of electrokinetic processing of dispersed materials is relatively high specific energy consumption per process; the dependence of specific 
energy costs on a large number of uncontrolled and difficultly controlled factors is due to the inadequacy of the existing theory and technology.

\section{MATHEMATICAL MODELING}

The need to solve the problem in the spatial coordinate system, corresponding to the real physical space, arises when the external force action and the axis of the capillary are mismatched, as well as with the harmonic external force, which is more adequate to the real world.

In general, the movement of moisture in a dispersed material can be expressed as the sum of a number of constituents, each of which shows the influence of a factor on electrokinetic energy-mass transfer (in this case electroosmosis). With the independent effect of these factors on energy costs in electroosmosis, there is:

$$
\begin{aligned}
& W_{\Sigma}=\Delta H m g+a_{1} \Delta B L \tau+a_{2} \Delta B L \tau+ \\
& k_{e} m_{e}+k_{g} m_{g}+k_{n} E^{2} S L+I^{2} R \tau+a_{3} E L \tau+ \\
& +a_{4} \tau \Delta T+a_{5} \tau \Delta T
\end{aligned}
$$

where

$\Delta H m g$ is the head loss;

$a_{1} \Delta B L \tau-$ loss of moisture conductivity;

$a_{2} \Delta B L \tau-$ loss of osmosis;

$k_{e} m_{e}-$ losses for electrolytic dissolution of electrodes;

$k_{g} m_{g}-$ losses for electrolytic gas formation;

$k_{n} E^{2} S L-$ loss of polarization of the dielectric of the dispersed phase;

$I^{2} R \tau$ - energy of electric heating;

$a_{5} E L \tau$ - work of thermal conductivity (thermoosmosis);

$$
\begin{aligned}
& a_{4} E L \tau-\text { work of difference media; } \\
& a_{3} \tau \Delta T-\text { work of electroosmosis. }
\end{aligned}
$$

The specific energy costs depend on each term of the equation. Of the listed phenomena and processes, only some perform useful work: the work of electroosmosis, the work of thermal conductivity; others are parasitic. Proceeding from the analysis of the physics of parasitic processes, the following working hypothesis is proposed:

A) Physical processes: loss of piezometric head and hydraulic head, loss of moisture conductivity, osmosis losses affect the energy of dehydration, but it is very difficult to isolate their effects.

B) Parasitic electrical processes make a decisive contribution to the total energy losses.
C) To eliminate the influence of parasitic electrical processes on energy consumption, it is sufficient to replace the treatment with a constant electric current for processing other types of electric energy, that is, to change the parameters of electricity.

In the absence of data on the energy relationship of processes in the general energy balance and the absence of boundary conditions for variables, an analytical solution of the system of equations is very difficult. The form of the energy diagram is shown in Fig. 3.

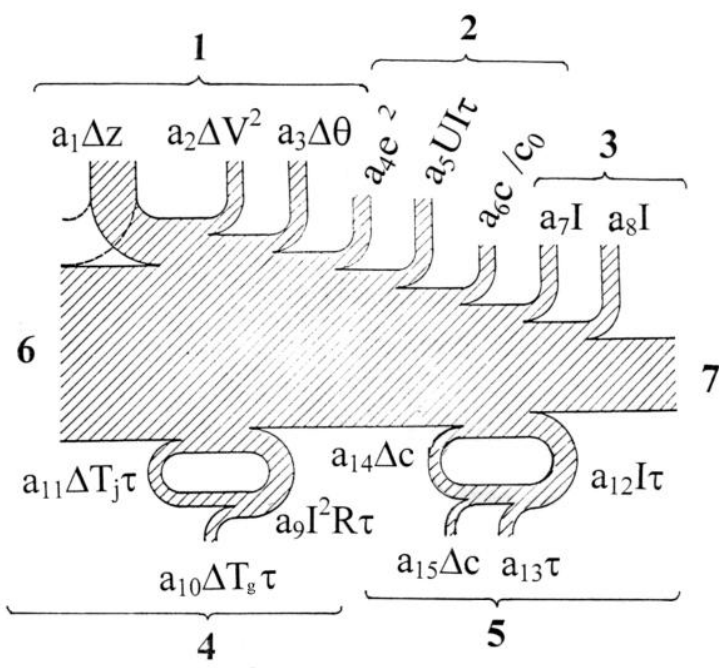

Fig. 3. Refined energy diagram of the process of electrokinetic dewatering of disperse material

In Figure 3: $a_{1} \Delta z$ - loss of piezometric head; $a_{2} \Delta V^{2}$ loss of frictional resistance; $a_{3} \Delta \theta$ - losses to overcome the forces of moisture conductivity; $a_{4} e^{2}$ - losses to overcome polarization of electrodes; $a_{5} U I \tau$ - losses to overcome the polarization of the dielectric of the disperse phase; $a_{6} c_{n} / c_{0}$ - losses to overcome the polarization of the electrolyte; $a_{7} I_{a}$ - losses for metal dissolution of electrodes; $a_{8} I_{a}$ - losses for electrolytic gas formation (decomposition of water); $a_{9} I_{a}^{2} R \tau$ - the energy of the electron-heating; $a_{10} \Delta T_{j} \tau$ - thermal losses to the external environment; $a_{11} \Delta T_{j} \tau$ - work of the forces of thermal conductivity; $a_{12} I_{a} \tau$ - work on the movement of ions of electrolytes losses to create a difference in concentrations; $a_{13} \tau$ - loss of osmosis due to the removal of electrolytes from the nearcathode space; $a_{14} \Delta c$ - the work of ionophoresis on the displacement of the dispersion medium - water; $a_{15} \Delta c$ loss of ionosmosis - counterflow of liquid; 1 macrophysical processes; 2 - electrophysical processes; 3 - 
electrochemical processes; 4 - work of electrokinetic energy and mass transfer; 5 - diffusion processes; 6 thermodiffusion processes; 7 - energy consumed from the source.

The type of energy flow $a_{1} \Delta z$ indicates that the piezometric head can create both a resistance to mass transfer in the main direction and an increase in the force of the electroosmotic pressure. This statement is confirmed experimentally [14] and is consistent with the theory.

The distribution of energy fluxes, characterizing thermodiffusion and electrodiffusion-phosphorous processes, indicates that only part of the energy of the electric will go into losses, while some will participate in the useful mass transfer in the form of thermal conductivity, ionosmosis and iontophoresis. Similarly, the distribution of energy flows of other diffusion processes.

The electrokinetic process in the energy aspect is a system of a series of physical, electrophysical, electrochemical, thermodiffusion and diffusion processes. A group of electrophysical and electrochemical processes are characterized by polarization at the molecular and ionic levels and electrolysis, which, when operating at a direct current, inevitably lead to excessive losses. The simplest solution for the removal of polarization at first glance is the transition to a variable asymmetric current.

With electroosmosis, a number of researchers note "orientational ordering of particles" and the formation of "chain structures" under the action of polarization forces. In addition, variable asymmetric nutrition should be affected by a decrease in the field-weakening effect of the relaxation of the diffuse layer and, as a consequence, an increase in the yield of water at the same energy costs.

In this case, it is useful to match the relaxation time of the double electric layer ( DEL) of the work material and the frequency of the electric field change to reduce the dielectric losses, the period of oscillations of the electric field strength should not exceed the relaxation time. Any polarization process is characterized by a finite relaxation time, so that at sufficiently high frequencies, when the field period is commensurable with the relaxation time, the phase shift between polarization and field becomes noticeable, which is manifested in dielectric losses. The relaxation time according to the theory of [15] for electrolytic polarization, that is, for the relaxation of the DEL, lies within 10-2 ... 10$4 \mathrm{sec}$. Experiments have shown that the least relaxation of the DEL affects frequencies $f=20 \ldots 320 \mathrm{~Hz}$.

In the process of operation of installations for electroosmotic dehydration of wet dispersed materials, the researchers observed "heating and drying of the soil adjacent to the anodes" [2]. Dehumidification of the material in the near-anodic region under the influence of electroosmosis leads to a significant increase in the resistivity of the material and to an increase in the voltage drop in the region adjacent to the anode. An increase in the voltage drop in turn leads to an increase in heating and an even greater desiccation of the material. Thus, near the anode, a progressively increasing process of overheating and drying out of the material, limiting the possibilities of the electroosmotic installation, can occur.

Hence, it can be concluded that the specific energy costs as the pre-anode zone drains increase. Switching the positive pole of the source from the anode to the anode, located on the line connecting the maximally remote anode with the cathode, should ensure the best possible mode of operation for each anode, since switching can be performed before the drained zone appears, thereby sharply reducing heat losses in the near-anode zone. In this case, it is useful to additionally measure the electric current through the material, and connect the anodes to the power source in series in the direction of the electric field, disconnecting the first anode from the positive pole of the electric power source and connecting the subsequent one taking into account the measured value of the electric current. The setting of the current relay should be such that the specific energy costs will be at an acceptable level from the point of view of the economy.

From the theory of continuum mechanics for a finite volume, it follows that electroosmosis is a process with diffusion, and the first component (the dispersion medium moisture) moves in one direction relative to the original volume, the second (the dispersed phase is a solid dielectric) into the other. Consequently, when studying the motion of multicomponent mixtures, it is necessary to combine the laws of continuum mechanics with the laws of physics and chemistry to change the mass of the component of the mixture per unit time per unit volume.

Consequently, the phenomenon of electrokinetics can not be considered in isolation from the continuum of mechanics, especially when working with closed volumes of processed materials, since it follows from the theory that the mass of each of the components in local volumes is subject to change and, therefore, the electrokinetic (electroosmosis coefficient), electric (specific electric conductivity) and electric power (specific energy consumption for moisture release) are characteristics of materials.

It should also be noted, that the theory of a continuum takes into account the motion of all components relative to the initial volume simultaneously - the superposition principle, which was not taken into account in previous work on electrokinetics, in connection with the fact that processes in unlimited volumes, dehydration of soils [2]. When the dispersed materials are dehydrated with electroosmosis in closed volumes, a persistent change in the physicochemical characteristics of the material takes place, which is manifested in an increase in the electrical resistivity of the material in the pre-anode zone and in the decrease in the cathode zone. As it is known, between the resistivity and the energy consumption, there is a stable interdependence. In practice, dehydration of the pre-anode zone leads to a loss of electrical conductivity of the preanode zone, an increase in specific energy inputs and, ultimately, an end to the dehydration process, until 
satisfactory characteristics of the work materials are obtained.

The greatest impact on the growth of energy costs in the process of electroosmotic dehydration in a closed volume is provided by the accelerated drainage of the near-anode zone and a jump in heat losses at the increased electric resistance of the near-anode zone, the relationship between the electrical resistivity and the moisture content of the material is obvious and experimentally proved. Moreover, the sharply identified drained pre-anode zone is not large - its thickness is usually within $0,05 \ldots 0,1 \mathrm{~m}$, but sufficient to interrupt the electric circuit. In this case, the main part of the supply voltage falls on the drained near-anode zone, which has a large electrical resistivity, and a small part for the rest of the material and the field strength in the material is insufficient to break loosely bound water, and the process of electroosmosis does not proceed. Electric energy is consumed only for heating the drained near-anodic zone. In order to move the loosely bound water, it is necessary to again create the electric field strength in the dehydrated mass above the threshold. This can be achieved in several ways: using the anode effect, switching the power supply from the anode to the anode - "running pulse", a short-time power source operation mode.

At the same time, specialists have the opinion that electroosmosis should be carried out at a constant current the logic of the work of electroosmotic pressure forces says exactly this, no one paid attention to the possibility of other phenomena, relaxation phenomena, occurring simultaneously with electroosmosis. Taking into account the phenomena that occur along with electroosmosis leads to the idea of dehydration on an alternating asymmetrical electric current. This allows one to remove the negative effect from the electric polarization of the electrodes [10], the effect of relaxation of the hydrostatic polarization of the dispersion medium is reduced by impulsive power supply [11], And the effect of the relaxation of the thermalconductivity forces is reduced by supplying the power supply with a "running pulse" [12]. In total, these three methods give a gain in the cost of electricity compared to electroosmosis at a constant current of up to 10-11 times.

\section{CONCLUSIONS}

1) It is established that the optimal physical process for dewatering the slurry can be an electroosmos.

2) In order to shorten the time of reclamation of slurry barns, it is advisable to remove the relaxation of various power gradient fields by changing the parameters of the electric power supplied to the electrodes.

3) It is planned to carry out the technological cycle of reclamation in one summer season, during which, with the help of electroosmosis, lower the slurry moisture and concentration of chemical contaminants to an acceptable level, and then plant the barn with siderates and thus return the territory to agricultural circulation.

\section{References}

[1] E. Y. Abramov, N. I. Schurov and M. V. Rozhkova, "Electric transport traction power supply system with distributed energy sources", IOP Conference Series: Materials Science and Engineering, 2016, Vol. 127, 7 p.

[2] E.Y. Abramov, A.A. Stang and S.A. Enkudinov, "Transformation of the urban electric transport system when using autonomous energy sources", Advanced Materials Research, 2014, Vol. 1040, pp. 778783.

[3] V.I. Sopov, N.I. Schurov, Y.A. Prokushev and A.A. Shtang, "Increasing the efficiency of the use of electrical energy in the subsubsystem of electric transport (Povishenie effektivnosti ispol'zovaniya elektricheskoy energii $\mathrm{V}$ subpodsisteme elektricheskogo transporta)", Improvement of technical means of electric transport (Sovershenstvovanie tehnicheskih sredstv elektricheskogo transporta), 2002, 189 p. [Digest of scientific works of the NSTU]

[4] B.A. Arzhannikov and A.A. Pyshkin, "Improving of DC power supply system based on automatic voltage regulation of traction substations (Sovershenstvovanie sistemi elektrosnabzheniya postoyannogo toka na osnove avtomaticheskogo regulirovaniya napryazheniya tyagovih podstanciy)", Ekaterinburg: "USURT Press", 2006, 116 p.

[5] V.I.Sopov, V.V. Biryukov, Y.A. Prokushev and Y.A. Rylov, "Analysis of power supply systems for rolling stock with various schemes of traction networks (Analiz sistem elektrosnabzheniya podvizhnogo sostava s razlichnimi shemami tyagovih setey)", Transport, Science, Engineering, Management (Transport, Nauka, Technika, Upravlenie). Vol. 2 (2008), pp. 49-53. [Digest of the VINITI RAS]

[6] V.V. Biryukov and A.V. Kulekina, "The calculation features of the electrical energy storage devices parameters in transport", The 11 International forum on strategic technology (IFOST 2016), pp. 41-43.

[7] N.I. Schurov, E.A. Spiridonov and A.V Larin, "Modes of traction power supply system in case of electric rolling stock equipped with energy storage", Applied Mechanics and Materials, 2014, Vol. 698, pp. 19-23.

[8] Photovoltaic (PV) research: National Renewable Energy Laboratory (NREL). Retrieved on http://www.nrel.gov/ncpv.

[9] N.W.A. Lidula and A.D. Rajapakse, "Microgrids research: a review of experimental microgrids and test systems", Renewable and Sustainable Energy Reviews, 2011, Vol. 15, pp. 186-202.

[10] B. Kroposki, R. Lasseter, T. Ise, S. Morozumi, S. Papathanassiou and N. Hatziargyriou, "A look at microgrid technologies and testing projects from around the world, making microgrids work", IEEE Power and Energy Magazine, 2008, Vol. 6, pp. 40-53.

[11] V.E. Rozenfeld, "Analytical calculation of electric railway networks (Analiticheskiy raschet setey elektricheskih zheleznih dorog)", The theoretical and scientific-practical peer-reviewed journal "Elektrichestvo", 1947, Vol. 9, pp. 6-17.

[12] V. G. Sysenko VG, D.A. Bosvit, E.N. Kosarev, "Improvement of the methodology for calculating the distributed traction power-supply system with an amplifying point", Power electronics and power engineering, vol. 9, pp. 8-18, 2014.

[13] O.I. Sablin, "Improving the efficiency of energy recovery in the electric transport system with limited traction power supply", Technological audit and production reserves, vol. 5/1, pp. 21-26, 2014.

[14] V.V. Biryukov, A.V. Kulekina, "The calculation features of the electrical energy storage devices parameters in transport", IFOST 2016, pp. 41-43, June 2016 [The 11 International forum on strategic technology, p. 596, 2016].

[15] V.I. Sopov, N.I. Schurov, Y.A. Prokushev and A.A. Shtang, "Increasing the efficiency of the use of electrical energy in the subsubsystem of electric transport", Improvement of technical means of electric transport, vol. 3 (29), pp. 115-123, Jule 2002 [Digest of scientific works of the NSTU, p. 189, 2002]. 